\title{
Análise combinatória de populações de milho pipoca em topcrosses
}

\author{
Adilson Luiz Seifert( ${ }^{(1)}$, Valéria Carpentieri-Pípolo(1), Josué Maldonado Ferreira ${ }^{(2)}$ e Antônio Carlos Gerage ${ }^{(3)}$
}

(1)Universidade Estadual de Londrina (UEL), Dep. de Agronomia, Rodovia Celso Garcia Cid, PR 445, Km 380, Campus Universitário, Caixa Postal 6.001, CEP 86051-990 Londrina, PR. E-mail: seifert@uel.br, pipolo@uel.br (2)UEL, Dep. de Biologia. E-mail: josuemf@uel.br (3)Instituto Agronômico do Paraná, Área de Melhoramento e Genética Vegetal, Caixa Postal 481, CEP 86001-970 Londrina, PR. E-mail: milhoger@pr.gov.br

\begin{abstract}
Resumo - O objetivo deste estudo foi avaliar, por meio de topcrosses, o potencial heterótico de 14 populações de milho pipoca. No ano agrícola 1997/1998, foram obtidos os híbridos topcrosses resultantes dos cruzamentos das populações com o testador, que se constituiu da mistura eqüitativa de sementes de todas as 14 populações. No ano agrícola 1999/2000, as populações e os híbridos foram avaliados em dois experimentos, em delineamento de blocos ao acaso, com três repetições. Foram estimados os parâmetros genéticos, e realizadas as análises de variância dos topcrosses e das populações. Com base nos resultados da capacidade geral de combinação das populações avaliadas nos topcrosses, para os caracteres produtividade e capacidade de expansão, foram selecionadas, para formar o composto amplo, as populações genitoras UEL SI, UEL PAP, UEL ZP, UEL PP, CMS 43 e RS 20. Esse composto apresenta maior potencial para uso como população base no melhoramento.
\end{abstract}

Termos para indexação: Zea mays everta, capacidade de expansão, heterose, capacidade geral de combinação.

\section{Combining ability analysis of popcorn populations in topcrosses}

\begin{abstract}
The objective of this work was to evaluate the heterotic potential of 14 popcorn populations through topcrosses. In the 1997/1998 crop year, topcrosses hybrids were obtained as a result of crosses between the populations and the tester, which was composed of an equal mixture of seeds from all 14 populations. The populations and the hybrids were evaluated in field, in a randomized block design, with three replications. The estimates for genetic parameters and the topcrosses variance analyses were made. Based on the results of yield and popping expansion of the experiments, the following populations were selected for the syntheses of composite: UEL SI, UEL PAP, UEL ZP, UEL PP, CMS 43 and RS 20. This broad composite is the most promising for use as base populations in breeding programs.
\end{abstract}

Index terms: Zea mays everta, popping expansion, heterosis, general combining ability.

\section{Introdução}

O milho pipoca, apesar da sua popularidade no Brasil, tem o processo de produção e comercialização pouco estudado. Há, também, escassez de informações oficiais sobre a área semeada, produtividade e quantidade importada. Estima-se que a produção nacional seja de aproximadamente 20 mil toneladas, e que o consumo atual esteja em torno de 81 mil toneladas de grãos (Galvão et al., 2000; Pereira \& Amaral Júnior, 2001).

Um dos maiores problemas enfrentados pelos produtores de milho pipoca é a falta de opções de cultivares no mercado, que reúnam boas características agronômicas com alto índice de capacidade de expansão. Segundo Sawazaki (1996), o fato de o produto nacional apresentar qualidade muito inferior ao importado está restringindo o mercado e desestimulando a cultura no Brasil. Esta situação ocorre em razão de a cultura do milho pipoca não ter recebido a mesma atenção que a cultura do milho comum. No Brasil, apenas sete cultivares híbridas de milho pipoca estão registradas no SNPC (Serviço Nacional de Proteção de Cultivares), das quais seis são de acesso restrito aos produtores parceiros das empresas detentoras de sementes (Sawazaki, 2001).

A heterose manifestada pelos híbridos pode, também, ser explorada em programas de melhoramento da cultura do milho pipoca. Nesse contexto, uma das técnicas genético-estatísticas mais interessante tem sido a análise de cruzamentos dialélicos, em razão do grande número de informações genéticas que podem oferecer ao melhorista. Um fator limitante à utilização de cruzamen- 
tos dialélicos é o número de genitores avaliados, particularmente nos casos em que o número de variedades (acessos) é grande, a ponto de comprometer a produção e avaliação dos híbridos, tornando mais adequada, nesse caso, a utilização dos topcrosses (Cruz \& Regazzi, 1994; Chaves \& Miranda Filho, 1997).

O trabalho realizado por Chaves \& Miranda Filho (1997) demonstrou que a predição da média de compostos pode ser feita sem o cruzamento dialélico das variedades genitoras. Os autores sugeriram o uso de topcrosses, em vez dos dialélicos, para a análise de variância e estimação dos parâmetros do modelo reduzido de Gardner (1967), e propuseram metodologia para a predição de médias de compostos.

O objetivo deste trabalho foi realizar a predição de médias de compostos de milho pipoca por meio de topcrosses, a fim de selecionar populações que possam ser utilizadas na síntese de compostos com alto rendimento de grãos e capacidade de expansão.

\section{Material e Métodos}

Foram utilizadas 14 populações de milho pipoca: nove procedentes de três gerações de seleção massal, estratificada dentro de variedades locais, selecionadas em lavouras de agricultores no Norte do Paraná (UEL MP, UEL YY, UEL SI, UEL MPS, UEL BG, UEL PAG, UEL PAP, UEL ZP e UEL PP); dois compostos procedentes da Embrapa Milho e Sorgo, Sete Lagoas, MG (CMS 42 e CMS 43); e três populações comerciais, cedidas pelo Instituto Agronômico do Paraná (RS 20/IPAGRO-RS, Capitão e Japonesa).

O campo para a obtenção dos cruzamentos foi instalado e conduzido em 1997/1998, na Área Experimental do Instituto Agronômico do Paraná (Iapar). As 14 populações foram semeadas separadamente, como fileiras femininas, e o testador como fileira masculina. $\mathrm{O}$ testador, uma mistura eqüitativa de sementes de todas as 14 populações, foi semeado em duas épocas, com intervalo de sete dias, intercalado com as fileiras femininas, na proporção de três para dois, respectivamente. As fileiras femininas e o testador foram semeados em linhas de $10 \mathrm{~m}$ de comprimento, com espaçamento de $0,9 \mathrm{~m}$ entre linhas e densidade de cinco plantas por metro. As fileiras femininas foram despendoadas no início do florescimento masculino.

As populações e os híbridos topcrosses foram avaliados em 1999/2000, em dois experimentos: Experimento I, instalado na Fazenda Experimental da Universidade Estadual de Londrina, PR, com semeadura realizada em novembro de 1999; Experimento II, ins- talado na Área Experimental do Iapar, com semeadura realizada em outubro de 1999. Os experimentos seguiram o delineamento de blocos ao acaso, com três repetições e 28 tratamentos (14 híbridos topcrosses e 14 populações).

A unidade experimental foi constituída de duas fileiras de $4 \mathrm{~m}$ de comprimento, espaçamento de $0,9 \mathrm{~m}$ entre fileiras e densidade de cinco plantas por metro linear. Os solos da área dos experimentos I e II foram classificados como Latossolo Vermelho distroférrico com relevo suave ondulado e textura muito argilosa.

As áreas utilizadas nesta pesquisa vêm sendo cultivadas, nos últimos 20 anos, com cultura anual, no sistema de rotação milho/soja, e a acidez do solo tem sido corrigida a cada dois anos, conforme exigências da análise de solo e recomendações técnicas.

A adubação empregada na semeadura, para ambos os ensaios, foi $500 \mathrm{~kg} \mathrm{ha}^{-1}$ da fórmula 8-28-16. Para a adubação de cobertura, utilizou-se $60 \mathrm{~kg} \mathrm{ha}^{-1}$ de uréia, 30 dias após a emergência (Instituto Agronômico de Campinas, 1996). Os demais tratos culturais foram realizados conforme as recomendações técnicas para a cultura do milho (Embrapa, 1996).

Foram avaliados os seguintes caracteres: altura de planta (AP, em metros), em três plantas competitivas por parcela; altura de espiga (AE, em metros); posição relativa da espiga na planta (PREP); número total de espigas colhidas por parcela (NE); número de plantas por parcela (ST); número de espigas por planta (NEP); florescimento feminino (FF); peso total de grãos na parcela (PG, kg parcela $\left.{ }^{-1}\right)$, corrigidos para umidade padrão de $13,5 \%$ e stand (ST) ideal de 40 plantas, por meio da metodologia de correção por covariância (Vencovsky \& Barriga, 1992); e capacidade de expansão (CE, em $\mathrm{mL} \mathrm{mL}^{-1}$ ), avaliada em grãos com $11 \%$ de umidade, em forno de microondas (Gökmen, 2004).

Para a análise dos dados, utilizou-se o SAS (SAS Institute, 1995). As estimativas dos parâmetros genéticos, efeito de variedades $\left(\mathrm{v}_{\mathrm{j}}\right)$, de heterose $\left(\mathrm{h}_{\mathrm{j}}\right)$, capacidade geral de combinação $\left(\mathrm{g}_{\mathrm{i}}\right)$ e as análises de variâncias de topcrosses, obtenção de $\hat{\mathrm{g}}_{\mathrm{i}}$ e $\mathrm{s}_{\mathrm{ij}}$, bem como k (tamanho do composto), para cada caráter avaliado, foram realizadas segundo Chaves \& Miranda Filho (1997), obtidas pelo modelo reduzido de Gardner (1967).

\section{Resultados e Discussão}

O volume de precipitações, em novembro, favoreceu o desenvolvimento e as avaliações dos híbridos topcrosses no experimento I. A estiagem ocorrida no mês de outubro prejudicou o experimento II, principal- 
mente nas avaliações de PG e CE. Assim, foi dada maior ênfase à discussão dos resultados obtidos no experimento I.

O resumo das análises de variância dos ensaios topcrosses, em dois experimentos para PG, NEP, AP, PREP e FF, é apresentado na Tabela 1. Pode-se observar que os coeficientes de variação (CV\%) foram baixos, o que revela boa precisão experimental para ensaios conduzidos em campo.

Foram detectadas diferenças significativas entre os tratamentos, para todos os caracteres avaliados (PG, NEP, AP, PREP e FF), exceto para CE no experimento I, e PREP no experimento II. No experimento II, exceto para NEP, foram detectados efeitos significativos de variedades $\left(\mathrm{v}_{\mathrm{j}}\right)$, para todos os caracteres com significância de tratamentos. Efeitos significativos de heterose média $(\bar{h})$ foram observados, no experimento I, para PG, CE, NEP, AP e FF, e para PG, NEP e AP no experimento II. Quando o quadrado médio da heterose média é significativo, a variância das freqüências gênicas entre as variedades é suficientemente grande em, pelo menos, parte dos locos com dominância, e as variedades, nessas condições, são divergentes nesses locos (Vencovsky, 1970).

O efeito de variedade $\left(\mathrm{v}_{\mathrm{j}}\right)$ está relacionado aos componentes aditivos das médias, e o da heterose $\left(\mathrm{h}_{\mathrm{j}}\right)$ rela- ciona-se aos componentes de dominância, o que indica uma diversidade entre os materiais, e diferenças no potencial dessas populações, para o uso em programas de melhoramento. Entretanto, para a heterose $\left(\mathrm{h}_{\mathrm{j}}\right)$, apenas os caracteres PG, NEP e FF, no experimento I, e FF no experimento II apresentaram efeitos significativos a 1 e $5 \%$ de probabilidade, respectivamente (Tabela 1). Para os caracteres AP e PREP, os efeitos de variedades $\left(\mathrm{V}_{\mathrm{j}}\right.$ 's $)$ foram preponderantes, o que indica a importância maior dos componentes aditivos sobre os de heterose $\left(h_{j}\right)$, na manifestação desses caracteres. Entretanto, para PG e FF, tanto efeitos aditivos como de dominância contribuíram para a manifestação dessas características. Essa situação pode ter sido decorrente do fato de as variedades não diferirem muito, em suas médias, para essas características.

A análise conjunta dos experimentos mostrou efeitos significativos para tratamento, em todos os caracteres avaliados (PG, NEP, AP, PREP e FF), o que mostra que os tratamentos apresentaram diferenças entre si. A significância para a interação tratamentos x local só foi verificada para os caracteres AP e PREP, indicando interação dos materiais com o ambiente para esses caracteres (Tabela 2). O efeito significativo de local revelou que o ambiente do experimento I favoreceu a maior expressão dos caracteres PG, AP, PREP e uma redução dos dias para florescimento.

Tabela 1. Análises de variância individuais de topcrosses de milho pipoca, em dois experimentos, para as características peso de grãos (PG, em t ha ${ }^{-1}$ ), capacidade de expansão (CE, em $\mathrm{mL} \mathrm{mL}^{-1}$ ), número de espiga por planta (NEP), altura de planta (AP, em metros), posição relativa da espiga na planta (PREP) e florescimento feminino (FF, em dias) ${ }^{(1)}$.

\begin{tabular}{|c|c|c|c|c|c|c|c|c|c|c|c|c|}
\hline \multirow[t]{2}{*}{ Fonte de variação } & \multirow[t]{2}{*}{ GL } & \multicolumn{10}{|c|}{ Quadrado médio } & \multirow[b]{2}{*}{$\mathrm{FF}$} \\
\hline & & PG & $\mathrm{CE}$ & NEP & $\mathrm{AP}$ & PREP & $\mathrm{FF}$ & PG & NEP & AP & PREP & \\
\hline & & \multicolumn{6}{|c|}{ - - } & & $-\mathrm{B}$ & perimentc & II --------- & 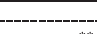 \\
\hline Tratamentos & 27 & $670,95^{* *}$ & $3,8502^{\mathrm{ns}}$ & $26,344^{* *}$ & $39,760^{* *}$ & $2,0495^{* *}$ & $24,451^{* *}$ & $171,51^{* *}$ & $25,231^{*}$ & $12,491^{* *}$ & $0,5420^{\mathrm{ns}}$ & $27,353^{* *}$ \\
\hline Variedades & 13 & $628,19^{* *}$ & $2,5129^{\mathrm{ns}}$ & $18,017_{* *}^{* *}$ & $51,982^{* *}$ & $3,3805^{* *}$ & $45,389^{* *}$ & $147,27^{* * *}$ & $17,461_{* *}^{\text {ns }}$ & $22,672^{* * *}$ & $0,7311^{\mathrm{ns}}$ & $48,465^{* *}$ \\
\hline Heterose média & 1 & $4.839,40_{* *}^{* *}$ & $16,3860^{*}$ & $196,440_{* * *}^{* *}$ & $288,730^{* *}$ & $0,8306^{\mathrm{ns}}$ & $7,682^{*}$ & $1.618,10^{* *}$ & $186,720^{* *}$ & $15,089^{* *}$ & $0,0167^{\text {ns }}$ & $0,321^{\text {ns }}$ \\
\hline Heterose de variedades & 13 & $393,06^{* *}$ & $4,2233^{\mathrm{ns}}$ & $21,587^{* *}$ & $8,386^{\mathrm{ns}}$ & $0,8124^{\text {ns }}$ & $4,803^{* *}$ & $84,48^{\text {ns }}$ & $20,578^{\text {ns }}$ & $2,109^{\mathrm{ns}}$ & $0,3933^{\text {ns }}$ & $8,319^{*}$ \\
\hline Erro & 54 & 148,33 & 2,7943 & 0,011 & 5,078 & 0,5562 & 1,218 & 57,58 & 12,580 & 1,761 & 0,5331 & 3,928 \\
\hline CV (\%) & & 15,67 & 7,17 & 0,30 & 3,74 & 3,84 & 1,91 & 13,50 & 10,36 & 2,84 & 4,27 & 3,10 \\
\hline
\end{tabular}

${ }^{(1)}$ Valores de quadrado médio multiplicados por 10 $0^{-3}$; graus de liberdade de erro igual a 53, para o caráter PG. nsNão-significativo. * e ** Significativo a 5 e $1 \%$ de probabilidade, respectivamente, pelo teste $\mathrm{F}$.

Tabela 2. Análises de variância conjuntas dos ensaios de topcrosses de milho pipoca, para as características peso de grãos (PG, em $t \mathrm{ha}^{-1}$ ), número de espiga por planta (NEP), altura de planta (AP, em metros), posição relativa da espiga na planta (PREP) e florescimento feminino (FF, em dias) ${ }^{(1)}$.

\begin{tabular}{|c|c|c|c|c|c|c|c|c|c|c|}
\hline \multirow[t]{3}{*}{ Caráter } & \multicolumn{10}{|c|}{ Fontes de variação } \\
\hline & \multicolumn{2}{|c|}{ Local } & \multicolumn{2}{|c|}{ Bloco/Local } & \multicolumn{2}{|c|}{ Tratamento } & \multicolumn{2}{|c|}{ Trat. x Local } & \multicolumn{2}{|c|}{ Erro } \\
\hline & GL & QM & GL & $\mathrm{QM}$ & GL & QM & GL & QM & GL & QM \\
\hline$P$ & 1 & $19.434,00^{\mathrm{ns}}$ & 2 & $406,15^{\mathrm{ns}}$ & 27 & $1.950,50^{* *}$ & 27 & $576,840^{\text {ns }}$ & 108 & 307,73 \\
\hline NEP & 1 & $4,53^{\text {ns }}$ & 2 & $112,23^{* * *}$ & 27 & $154,65_{* * *}^{* *}$ & 27 & $0,078^{\mathrm{ns}}$ & 110 & 20,70 \\
\hline $\mathrm{AP}$ & 1 & $7.654,40^{* *}$ & 2 & $78,44^{* *}$ & 27 & $129,20^{* *}$ & 27 & $27,548^{* *}$ & 110 & 10,63 \\
\hline PREP & 1 & $238,35^{* *}$ & 2 & $6,70^{*}$ & 27 & $4,91^{* *}$ & 27 & $2,855^{*}$ & 110 & 1,72 \\
\hline $\mathrm{FF}$ & 1 & $1.615,70^{* *}$ & 2 & $20,79^{\mathrm{ns}}$ & 27 & $146,31^{* *}$ & 27 & $9,103^{\text {ns }}$ & 110 & 7,72 \\
\hline
\end{tabular}

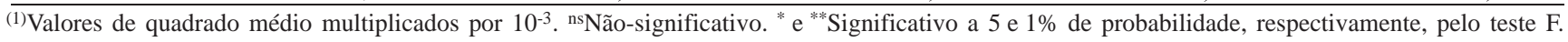


As estimativas positivas de $v_{j}$, $h_{j}$ e $g_{i}$ são apresentadas na Tabela 3. Valores de gi positivos, para PG, foram observados em UEL YY, UEL SI, UEL PAP, UEL ZP, UEL PP, CMS 42, CMS 43 e RS 20, no experimento I. Esses valores foram confirmados no experimento II, para UEL MP, UEL MPS, UEL PAG, UEL PAP, UEL PP, CMS 42, CMS 43 e Japonesa. Estas populações apresentaram as maiores estimativas positivas de $g_{i}$, sendo, portanto, de maior potencial para aumentar a média desse caráter, quando utilizadas em cruzamentos. Estes dados são demonstrados nas Tabelas 4 e 5, experimentos I e II, respectivamente, que apresentam tais populações como as melhores médias topcrosses.

A população CMS 43 apresentou o melhor desempenho na avaliação per se e também em combinação híbrida com o testador, comprovando os valores positivos de $v_{j}$ e $g_{i}$ (Tabela 4). Entretanto, nem sempre as populações que se destacam na avaliação per se são as que conseguem bons desempenhos quando em combinações híbridas (Hallauer, 1990).

Para CE, os maiores valores positivos de $\mathrm{v}_{\mathrm{j}}$ foram estimados para as populações CMS 43, UEL YY, Japonesa, UEL SI, UEL ZP, UEL PP e Capitão. Para $h_{j}$, os maiores valores positivos ocorreram para as populações RS 20, UEL PAG, UEL MPS, UEL PAP, UEL MP, UEL BG e UEL ZP. Para $g_{i}$, as populações RS 20, UEL MPS, UEL PAG, UEL ZP, UEL PAP, CMS 43, UEL MP, UEL BG e UEL PP apresentaram maiores valores positivos.

A capacidade de expansão (CE) é o caráter de maior importância no milho pipoca, por estar relacionado diretamente com a qualidade da pipoca. As populações com maiores efeitos de $v_{j}$ (Tabela 3), que apresentaram as maiores médias da variável CE (Tabela 4), possuem maiores freqüências de alelos favoráveis para CE, provavelmente como resultado do melhoramento para esta característica. Neste trabalho, a média geral das populações para CE foi $24,10 \mathrm{~mL} \mathrm{~mL}^{-1}$ (Tabela 4). A média geral da capacidade de expansão dos materiais comerciais testados no Ensaio Nacional de Milho Pipoca 1991/1992, realizado pela Embrapa, foi de 17,39 (Pacheco, 1992), muito abaixo do padrão exigido pelas empresas que atuam no setor. Em 1997/1998, em Londrina, PR, diversos genótipos avaliados por Carpentieri-Pípolo et al. (2002) apresentaram a CE entre 18,53 e 27,50 mL mL-1. Destacaram-se os genótipos UEL ZP, UEL SI e UEL PAP, com valores para CE de 27,50, 27,15 e 24,40 $\mathrm{mL} \mathrm{mL}^{-1}$, respectivamente. Tais resultados são considerados promissores para utilização dos materiais em programas de melhoramento.

A seleção para maiores valores médios de CE (Tabela 4) acarretou diminuição dos potenciais produtivos das populações. Isto era previsto, em virtude da existência de correlação negativa entre estas características (Lyerly, 1942; Lira, 1983; Silva et al., 1993; Sawazaki, 1996; Carpentieri-Pípolo et al., 2002). Todavia, as relações não são grandes o bastante para impedir que se obtenham ganhos positivos para CE e produção, simultaneamente, quando se faz a seleção com base nestes dois caracteres (Sawazaki, 1995; Carpentieri-Pípolo et al., 2002).

Os resultados médios observados para o caráter NEP, nas populações e topcrosses para os experimentos I e II, são apresentados nas Tabelas 4 e 5, respectivamente. Em geral, as médias observadas foram de aproximadamente uma espiga por planta. Destacaram-se com os maiores valores médios a população parental CMS 43 e o topcross Japonesa TC. Galvão et al. (2000) relataram relação direta entre prolificidade e produtividade, com magnitude de correlação de 0,65 para esses caracteres.

Tabela 3. Estimativas dos efeitos de variedade $\left(\mathrm{v}_{\mathrm{j}}\right)$, de heterose $\left(\mathrm{h}_{\mathrm{j}}\right)$ e da capacidade geral de combinação $\left(\mathrm{g}_{\mathrm{i}}\right)$, obtidas nos experimentos de avaliação de topcrosses de milho pipoca ${ }^{(1)}$.

\begin{tabular}{|c|c|c|c|c|c|c|c|c|c|c|c|c|c|c|c|c|c|c|c|c|c|}
\hline \multirow[t]{3}{*}{ População } & \multicolumn{12}{|c|}{ Experimento I } & \multicolumn{9}{|c|}{ Experimento II } \\
\hline & \multicolumn{3}{|c|}{ PG } & \multicolumn{3}{|c|}{$\mathrm{CE}$} & \multicolumn{3}{|c|}{ AP } & \multicolumn{3}{|c|}{$\mathrm{FF}$} & \multicolumn{3}{|c|}{ PG } & \multicolumn{3}{|c|}{$\mathrm{AP}$} & \multicolumn{3}{|c|}{$\mathrm{FF}$} \\
\hline & $v_{i}$ & $\mathrm{~h}_{\mathrm{i}}$ & $\mathrm{g}_{\mathrm{i}}$ & $\mathrm{v}_{\mathrm{i}}$ & $h_{j}$ & $\mathrm{~g}_{\mathrm{i}}$ & $\mathrm{v}_{\mathrm{i}}$ & $h_{j}$ & $\mathrm{~g}_{\mathrm{i}}$ & $\mathrm{v}_{\mathrm{i}}$ & $h_{j}$ & $\mathrm{~g}_{\mathrm{i}}$ & $\mathrm{v}_{\mathrm{i}}$ & $h_{j}$ & $\mathrm{~g}_{\mathrm{i}}$ & $\mathrm{v}_{\mathrm{i}}$ & $\mathrm{h}_{\mathrm{j}}$ & $\mathrm{g}_{\mathrm{i}}$ & $\mathrm{v}_{\mathrm{i}}$ & $h_{j}$ & $\mathrm{~g}_{\mathrm{i}}$ \\
\hline UEL MP & 0,63 & $-1,33$ & $-1,02$ & $-1,83$ & 1,54 & 0,63 & 0,13 & 0,01 & 0,16 & $-1,24$ & 1,94 & 1,32 & 0,30 & $-0,09$ & 0,06 & 0,13 & $-0,07$ & $-0,01$ & 1,93 & 2,93 & 3,89 \\
\hline UEL YY & $-1,10$ & 0,79 & 0,2 & 2,25 & $-3,08$ & $-1,9$ & $-0,19$ & 0,05 & -0 & -( & 0,78 & 0,49 & 22 & $-0,08$ & 19 & 5 & 07 & -0 & 07 & 93 & 89 \\
\hline UEL & 0,34 & 0,47 & 0,6 & 1,54 & $-2,03$ & $-1,23$ & 0,06 & 0,06 & $-0,03$ & 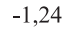 & 1,17 & 0 & $-0,14$ & 0,00 & -0 & 0,03 & 0,02 & 4 &, 74 & 1,76 & ,39 \\
\hline UEL & 0,04 & $-1,28$ & $-1,26$ & $-0,46$ & 3,12 & 2,8 & $-0,17$ & $-0,14$ & $-0,23$ & -4 & 0,97 & -1 , & $-0,01$ & 0,03 & 0 & $-0,10$ & $-0,05$ & -0 & $-4,74$ & $-0,96$ & $-3,33$ \\
\hline UEL BG & $-0,55$ & $-0,01$ & $-0,28$ & $-1,29$ & 1,23 & 0,58 & $-0,17$ & 0,10 & 0,01 & $-0,24$ & $-0,58$ & $-0,70$ & 0,03 & $-0,45$ & $-0,44$ & $-0,07$ & 0,02 & $-0,01$ & $-3,07$ & 3,12 & 1,59 \\
\hline UEL PAG & 0,12 & $-0,26$ & $-0,20$ & $-3,67$ & 3,29 & 1,46 & 0,02 & $-0,12$ & $-0,11$ & $-4,90$ & 5,25 & 2,80 & 0,43 & 0,05 & 0,27 & 0,00 & 0,05 & 0,06 & $-3,40$ & 2,93 & 1,23 \\
\hline UEL PAP & $-0,96$ & 0,59 & 0,11 & $-1,58$ & 2,03 & 1,24 & $-0,17$ & $-0,06$ & $-0,15$ & $-4,57$ & 0,78 & $-1,51$ & $-0,19$ & 0,28 & 0,18 & $-0,17$ & 0,08 & $-0,01$ & $-5,40$ & $-1,35$ & $-4,05$ \\
\hline UEL ZP & 0,06 & 0,17 & 0,20 & 1,54 & 0,50 & 1,27 & $-0,02$ & 0,01 & 0,00 & $-0,24$ & 1,75 & 1,63 & $-0,22$ & $-0,14$ & $-0,25$ & 0,06 & $-0,03$ & $-0,01$ & $-1,40$ & 1,76 & 1,06 \\
\hline UEL PP & 0,24 & 0,21 & 0,33 & 1,08 & $-0,26$ & 0,28 & 0,13 & $-0,05$ & 0,02 & $-1,24$ & 1,17 & 0,55 & 0,22 & 0,19 & 0,30 & $-0,07$ & $-0,04$ & $-0,07$ & $-2,74$ & 0,21 & $-1,16$ \\
\hline Capitão & 0,01 & $-1,59$ & $-1,57$ & 0,33 & $-4,14$ & $-3,98$ & $-0,15$ & $-0,12$ & $-0,20$ & $-0,24$ & $-6,81$ & $-6,92$ & $-0,29$ & $-0,42$ & $-0,56$ & $-0,14$ & $-0,09$ & $-0,16$ & $-4,07$ & $-2,51$ & $-4,55$ \\
\hline CMS 042 & 0,99 & 0,66 & 1,16 & $-1,25$ & $-1,52$ & $-2,15$ & 0,31 & 0,25 & 0,40 & 0,76 & $-0,78$ & $-0,40$ & 0,32 & 0,03 & 0,19 & 0,26 & $-0,04$ & 0,09 & 3,93 & 1,76 & 3,73 \\
\hline CMS 043 & 1,27 & 0,73 & 1,37 & 2,37 & $-0,23$ & 0,95 & 0,36 & 0,01 & 0,28 & 2,76 & $-3,11$ & $-1,73$ & 0,76 & $-0,23$ & 0,15 & 0,23 & $-0,02$ & 0,10 & 4,93 & $-11,26$ & $-8,80$ \\
\hline & $-0,40$ & 0,20 & 0,00 & 1,62 & $-4,17$ & $-3,36$ & $-0,10$ & 0,10 & 0,05 & 20,76 & $-3,11$ & 7,27 & $-0,67$ & 1,10 & 0,77 & 0,08 & 0,08 & 0,13 & 18,93 & 0,40 & 9,87 \\
\hline RS 20 & $-0,69$ & 0,63 & 0,28 & $-0,67$ & 3,73 & 3,40 & $-0,05$ & $-0,15$ & $-0,17$ & $-5,57$ & 0,58 & $-2,20$ & $-0,31$ & $-0,27$ & $-0,42$ & $-0,07$ & 0,02 & $-0,01$ & $-2,07$ & $-1,74$ & $-2,77$ \\
\hline
\end{tabular}

(1)PG: peso de grãos (t ha-1); CE: capacidade de expansão ( $\left.\mathrm{mL} \mathrm{mL}^{-1}\right)$; AP: altura de planta (m); FF: florescimento feminino (dias). 
Para os caracteres AP e FF, valores negativos e menores para estimativas de parâmetros são altamente desejáveis, pois contribuem, por meio do modelo aditivo, na redução da média de AP e FF das populações.

Exceto para $h_{j}$ no experimento II, as populações CMS 42 e CMS 43 apresentaram valores de $v_{j}$, $h_{j}$ e $\mathrm{g}_{\mathrm{i}}$ positivos, o que contribuiu para o aumento da AP, provavelmente por possuirem maior freqüência de genes aditivos (Tabela 3). Entretanto, as populações RS 20 e UEL SI, no experimento I, e UEL PAP e UEL MP, no experimento II, apresentaram gi's negativos, que podem auxiliar na redução da AP (Tabela 3). Estes dados estão nas Tabelas 4 e 5, experimentos I e II, respectivamente, em que estas populações apresentam as melhores médias per se e em combinação híbrida com o testador, tanto para aumentar quanto para reduzir a AP.

Nos dados de progênies de irmãos germanos, a variedade Santa Rosa, obtida a partir de materiais america-

Tabela 4. Médias das populações e respectivos cruzamentos topcrosses, obtidos do experimento de milho pipoca na UEL ${ }^{(1)}$.

\begin{tabular}{|c|c|c|c|c|c|c|}
\hline \multirow[t]{2}{*}{ População ${ }^{(2)}$} & \multicolumn{6}{|c|}{ Experimento I } \\
\hline & $\mathrm{PG}$ & $\mathrm{CE}$ & NEP & AP & PREP & FF \\
\hline CMS 043 TC & $4,14 a$ & $23,5 a$ & $1,29 \mathrm{c}$ & $2,27 \mathrm{a}$ & $0,62 b$ & $57 \mathrm{c}$ \\
\hline CMS 042 TC & $3,94 \mathrm{a}$ & $20,6 \mathrm{a}$ & $1,07 \mathrm{i}$ & $2,37 \mathrm{a}$ & $0,66 \mathrm{a}$ & $58 \mathrm{c}$ \\
\hline UEL SI TC & $3,45 \mathrm{a}$ & $21,6 \mathrm{a}$ & $1,06 \mathrm{i}$ & $1,99 b$ & $0,62 b$ & $59 c$ \\
\hline CMS 043 & $3,31 \mathrm{a}$ & $26,5 \mathrm{a}$ & $1,26 \mathrm{~d}$ & $2,16 \mathrm{a}$ & $0,66 \mathrm{a}$ & $60 c$ \\
\hline UEL PP TC & $3,18 \mathrm{a}$ & $22,9 a$ & $1,20 \mathrm{f}$ & $2,03 \mathrm{~b}$ & $0,64 \mathrm{a}$ & $59 c$ \\
\hline RS $20 \mathrm{TC}$ & $3,07 \mathrm{a}$ & $25,4 a$ & $1,15 \mathrm{~g}$ & $1,85 \mathrm{c}$ & $0,62 b$ & $56 c$ \\
\hline UEL ZP TC & $3,05 \mathrm{a}$ & $23,8 \mathrm{a}$ & $1,02 \mathrm{k}$ & $2,01 b$ & $0,63 \mathrm{a}$ & $60 c$ \\
\hline CMS 042 & $3,03 \mathrm{a}$ & $22,8 \mathrm{a}$ & $0,97 \mathrm{~m}$ & $2,12 \mathrm{a}$ & $0,66 \mathrm{a}$ & $58 \mathrm{c}$ \\
\hline UEL YY TC & $3,00 \mathrm{a}$ & $21,0 \mathrm{a}$ & $1,04 \mathrm{j}$ & $1,96 \mathrm{~b}$ & $0,56 \mathrm{~b}$ & $59 c$ \\
\hline UEL PAP TC & $2,90 \mathrm{a}$ & $23,5 \mathrm{a}$ & $1,09 \mathrm{~h}$ & $1,87 \mathrm{c}$ & $0,54 \mathrm{~b}$ & $57 \mathrm{c}$ \\
\hline Japonesa TC & $2,85 \mathrm{a}$ & $19,8 \mathrm{a}$ & $1,48 \mathrm{a}$ & $2,04 b$ & $0,68 \mathrm{a}$ & $66 \mathrm{~b}$ \\
\hline UEL PAG TC & $2,72 \mathrm{a}$ & $23,5 \mathrm{a}$ & $1,40 \mathrm{~b}$ & $1,91 b$ & $0,62 b$ & $60 c$ \\
\hline UEL MP & $2,67 \mathrm{a}$ & $22,3 a$ & $1,09 \mathrm{~h}$ & $1,94 b$ & $0,61 b$ & $56 c$ \\
\hline UEL BG TC & $2,59 \mathrm{a}$ & $23,0 \mathrm{a}$ & $0,96 \mathrm{~m}$ & $2,00 \mathrm{~b}$ & $0,57 \mathrm{~b}$ & $58 \mathrm{c}$ \\
\hline UEL SI & $2,39 b$ & $25,6 a$ & 0,860 & $1,86 \mathrm{c}$ & $0,59 \mathrm{~b}$ & $56 c$ \\
\hline UEL PP & $2,28 b$ & $25,2 \mathrm{a}$ & $1,15 \mathrm{~g}$ & $1,94 b$ & $0,61 b$ & $56 c$ \\
\hline UEL PAG & $2,17 b$ & $20,4 a$ & 1,001 & $1,83 \mathrm{c}$ & $0,54 b$ & $52 \mathrm{~d}$ \\
\hline UEL ZP & $2,10 \mathrm{~b}$ & $25,6 \mathrm{a}$ & $0,96 \mathrm{~m}$ & $1,79 \mathrm{c}$ & $0,58 \mathrm{~b}$ & $57 \mathrm{c}$ \\
\hline UEL MPS & $2,08 \mathrm{~b}$ & $23,6 a$ & 1,001 & $1,64 d$ & $0,58 \mathrm{~b}$ & $53 \mathrm{~d}$ \\
\hline Capitão & $2,05 b$ & $24,4 a$ & $0,88 \mathrm{n}$ & $1,65 \mathrm{~d}$ & $0,64 \mathrm{a}$ & $57 \mathrm{c}$ \\
\hline UEL MP TC & $2,05 b$ & $23,0 \mathrm{a}$ & $1,23 \mathrm{e}$ & $2,16 \mathrm{a}$ & $0,67 \mathrm{a}$ & $59 c$ \\
\hline UEL MPS TC & $1,80 \mathrm{~b}$ & $25,0 \mathrm{a}$ & $1,16 \mathrm{~g}$ & $1,80 \mathrm{c}$ & $0,60 \mathrm{~b}$ & $57 \mathrm{c}$ \\
\hline Japonesa & $1,64 b$ & $25,7 \mathrm{a}$ & $0,73 p$ & $1,70 \mathrm{~d}$ & $0,73 \mathrm{a}$ & $78 \mathrm{a}$ \\
\hline Capitão TC & $1,52 b$ & $19,2 \mathrm{a}$ & $1,04 j$ & $1,82 \mathrm{c}$ & $0,67 \mathrm{a}$ & $52 \mathrm{~d}$ \\
\hline UEL BG & $1,49 b$ & $22,8 \mathrm{a}$ & $1,02 \mathrm{k}$ & $1,63 \mathrm{~d}$ & $0,59 \mathrm{~b}$ & $57 \mathrm{c}$ \\
\hline RS 20 & $1,35 b$ & $23,4 a$ & $1,03 \mathrm{j}$ & $1,75 \mathrm{c}$ & $0,60 \mathrm{~b}$ & $52 \mathrm{~d}$ \\
\hline UEL PAP & $1,08 b$ & $22,5 \mathrm{a}$ & $0,89 n$ & $1,64 d$ & $0,55 \mathrm{~b}$ & $53 \mathrm{~d}$ \\
\hline UEL YY & $0,94 b$ & $26,3 \mathrm{a}$ & 1,001 & $1,61 \mathrm{~d}$ & $0,60 \mathrm{~b}$ & $57 \mathrm{c}$ \\
\hline Média populações & 2,04 & 24,10 & 0,99 & 1,80 & 0,61 & 57 \\
\hline Média topcrosses & 2,87 & 22,55 & 1,16 & 2,01 & 0,62 & 58 \\
\hline Média geral & 2,46 & 23,32 & 1,07 & 1,90 & 0,62 & 58 \\
\hline $\mathrm{CV}(\%)$ & 26,89 & 12,41 & 0,61 & 6,48 & 6,62 & 3,31 \\
\hline
\end{tabular}

(1)PG: peso de grãos ( $\left.\mathrm{t} \mathrm{ha}^{-1}\right)$; $\mathrm{CE}$ : capacidade de expansão $\left(\mathrm{mL} \mathrm{mL}^{-1}\right)$; NEP: número de espigas por planta; AP: altura de planta (m); PREP: posição relativa da espiga na planta (m); FF: florescimento feminino (dias); médias seguidas da mesma letra, na coluna, não diferem entre si pelo teste de Scott-Knott a 5\% de probabilidade. ${ }^{(2)} \mathrm{TC}$ : cruzamentos topcrosses. nos, obteve médias de 1,62 m para AP (Regitano Neto, 1993). Menor porte de planta (1,67 m) também foi obtido por Lira (1983), em uma população de Lavras. Populações de porte alto são suscetíveis ao acamamento e quebramento, e populações de porte baixo, além da menor suscetibilidade a estes problemas, possuem melhor adaptação à mecanização durante a colheita, melhor adaptabilidade à semeadura, em densidades populacionais mais altas, e maior resistência ao estresse hídrico (Santacruz-Varela et al., 2004).

Os maiores valores positivos de $v_{j}$ e $g_{i}$ para FF foram apresentados pela população Japonesa, nos dois experimentos (Tabela 3). Estes resultados indicam que, em termos práticos, ocorreu um aumento geral de 20 dias para $\mathrm{FF}$, com relação à média geral dos experimentos I e II (Tabelas 4 e 5). Neste trabalho considerou-se que, pelo conjunto de suas características, a população Japonesa pertence a um grupo heterótico diferente das demais populações.

Tabela 5. Médias das populações e respectivos cruzamentos topcrosses, obtidos do experimento de milho pipoca no $\operatorname{IAPAR}^{(1)}$.

\begin{tabular}{lccccc}
\hline População $^{(2)}$ & \multicolumn{5}{c}{ Experimento II } \\
\cline { 2 - 6 } & PG & NEP & AP & PREP & FF \\
\hline Japonesa TC & $2,63 \mathrm{a}$ & $1,49 \mathrm{a}$ & $1,61 \mathrm{a}$ & $0,57 \mathrm{a}$ & $74 \mathrm{~b}$ \\
CMS 043 & $2,29 \mathrm{a}$ & $1,27 \mathrm{a}$ & $1,68 \mathrm{a}$ & $0,52 \mathrm{a}$ & $69 \mathrm{c}$ \\
UEL PP TC & $2,29 \mathrm{a}$ & $1,20 \mathrm{a}$ & $1,43 \mathrm{c}$ & $0,55 \mathrm{a}$ & $62 \mathrm{~d}$ \\
UEL PAG TC & $2,28 \mathrm{a}$ & $1,41 \mathrm{a}$ & $1,55 \mathrm{~b}$ & $0,55 \mathrm{a}$ & $65 \mathrm{c}$ \\
CMS 042 TC & $2,21 \mathrm{a}$ & $1,07 \mathrm{a}$ & $1,60 \mathrm{a}$ & $0,57 \mathrm{a}$ & $67 \mathrm{c}$ \\
CMS 043 TC & $2,20 \mathrm{a}$ & $1,30 \mathrm{a}$ & $1,60 \mathrm{a}$ & $0,52 \mathrm{a}$ & $57 \mathrm{~d}$ \\
UEL PAP TC & $2,16 \mathrm{a}$ & $1,09 \mathrm{a}$ & $1,48 \mathrm{~b}$ & $0,52 \mathrm{a}$ & $60 \mathrm{~d}$ \\
UEL MP TC & $2,09 \mathrm{a}$ & $1,24 \mathrm{a}$ & $1,50 \mathrm{~b}$ & $0,58 \mathrm{a}$ & $67 \mathrm{c}$ \\
UEL MPS TC & $2,04 \mathrm{a}$ & $1,15 \mathrm{a}$ & $1,40 \mathrm{c}$ & $0,56 \mathrm{a}$ & $61 \mathrm{~d}$ \\
UEL PAG & $1,97 \mathrm{a}$ & $1,02 \mathrm{a}$ & $1,45 \mathrm{c}$ & $0,54 \mathrm{a}$ & $61 \mathrm{~d}$ \\
UEL SI TC & $1,95 \mathrm{a}$ & $1,07 \mathrm{a}$ & $1,53 \mathrm{~b}$ & $0,48 \mathrm{a}$ & $65 \mathrm{c}$ \\
CMS 042 & $1,88 \mathrm{a}$ & $0,98 \mathrm{a}$ & $1,72 \mathrm{a}$ & $0,56 \mathrm{a}$ & $68 \mathrm{c}$ \\
UEL YY TC & $1,84 \mathrm{a}$ & $1,05 \mathrm{a}$ & $1,48 \mathrm{~b}$ & $0,54 \mathrm{a}$ & $65 \mathrm{c}$ \\
UEL MP & $1,83 \mathrm{a}$ & $1,10 \mathrm{a}$ & $1,58 \mathrm{a}$ & $0,58 \mathrm{a}$ & $66 \mathrm{c}$ \\
UEL ZP TC & $1,78 \mathrm{a}$ & $1,03 \mathrm{a}$ & $1,50 \mathrm{~b}$ & $0,55 \mathrm{a}$ & $65 \mathrm{c}$ \\
UEL PP & $1,76 \mathrm{a}$ & $1,16 \mathrm{a}$ & $1,38 \mathrm{c}$ & $0,53 \mathrm{a}$ & $61 \mathrm{~d}$ \\
UEL BG TC & $1,65 \mathrm{~b}$ & $0,97 \mathrm{a}$ & $1,48 \mathrm{~b}$ & $0,55 \mathrm{a}$ & $65 \mathrm{c}$ \\
RS 20 TC & $1,63 \mathrm{~b}$ & $1,16 \mathrm{a}$ & $1,48 \mathrm{~b}$ & $0,52 \mathrm{a}$ & $61 \mathrm{~d}$ \\
UEL BG & $1,56 \mathrm{~b}$ & $1,02 \mathrm{a}$ & $1,38 \mathrm{c}$ & $0,53 \mathrm{a}$ & $61 \mathrm{~d}$ \\
UEL MPS & $1,52 \mathrm{~b}$ & $1,02 \mathrm{a}$ & $1,35 \mathrm{c}$ & $0,56 \mathrm{a}$ & $59 \mathrm{~d}$ \\
Capitão TC & $1,52 \mathrm{~b}$ & $1,05 \mathrm{a}$ & $1,35 \mathrm{c}$ & $0,53 \mathrm{a}$ & $60 \mathrm{~d}$ \\
UEL SI & $1,40 \mathrm{~b}$ & $0,86 \mathrm{a}$ & $1,48 \mathrm{~b}$ & $0,52 \mathrm{a}$ & $63 \mathrm{~d}$ \\
UEL PAP & $1,34 \mathrm{~b}$ & $0,89 \mathrm{a}$ & $1,28 \mathrm{c}$ & $0,53 \mathrm{a}$ & $59 \mathrm{~d}$ \\
UEL YY & $1,32 \mathrm{~b}$ & $1,01 \mathrm{a}$ & $1,30 \mathrm{c}$ & $0,51 \mathrm{a}$ & $62 \mathrm{~d}$ \\
UEL ZP & $1,32 \mathrm{~b}$ & $0,97 \mathrm{a}$ & $1,52 \mathrm{~b}$ & $0,54 \mathrm{a}$ & $63 \mathrm{~d}$ \\
Capitão & $1,30 \mathrm{~b}$ & $0,90 \mathrm{a}$ & $1,32 \mathrm{c}$ & $0,57 \mathrm{a}$ & $60 \mathrm{~d}$ \\
RS 20 & $1,23 \mathrm{~b}$ & $1,04 \mathrm{a}$ & $1,38 \mathrm{c}$ & $0,54 \mathrm{a}$ & $62 \mathrm{~d}$ \\
Japonesa & $0,87 \mathrm{~b}$ & $0,76 \mathrm{a}$ & $1,53 \mathrm{~b}$ & $0,55 \mathrm{a}$ & $83 \mathrm{a}$ \\
\hline Média populações & 1,54 & 1,00 & 1,45 & 0,54 & 64 \\
Média topcrosses & 2,02 & 1,16 & 1,50 & 0,54 & 64 \\
Média geral & 1,78 & 1,08 & 1,48 & 0,54 & 64 \\
\hline CV (\%) & 23,16 & 17,85 & 4,92 & 7,39 & 5,37 \\
\hline
\end{tabular}

(1)PG: peso de grãos ( $\left.\mathrm{t} \mathrm{ha}^{-1}\right)$; NEP: número de espigas por planta; AP: altura de planta (m); PREP: posição relativa da espiga na planta (m); FF: florescimento feminino (dias). Médias seguidas da mesma letra, na coluna, não diferem entre si pelo teste de Scott-Knott a 5\% de probabilidade. (2)TC: cruzamentos topcrosses. 
O desempenho médio dos topcrosses foi superior ao obtido pelas populações per se, e evidenciou a ocorrência de heterose nos topcrosses. Alguns melhoristas acreditam que as avaliações de populações em topcrosses são prejudicadas pelo fato de os cruzamentos apresentarem diversas características indesejáveis, associadas à introdução dos alelos do testador (Lonnquist \& Lindsey, 1964; Souza, 2000). Teoricamente, o testador contribui com 50\% dos alelos, e apenas os 50\% restantes representariam os genótipos avaliados (Pinto et al., 2004). Além deste efeito, os alelos do testador podem interagir ou não com os alelos dos genótipos a serem avaliados, prejudicando a representatividade das diferenças desses genótipos. No presente trabalho, o fato de o testador ser constituído por uma mistura das sementes das populações não influenciou na discriminação dos topcrosses.

Segundo Ferreira (1999), os efeitos heteróticos também são aproveitados na síntese de compostos. Por isso, o parâmetro $\mathrm{g}_{\mathrm{i}}$ fornece um bom indicativo da contribui- ção da população na formação do composto, pois permite o cruzamento e recombinação de materiais com elevada freqüência de alelos favoráveis.

Nas 14 populações avaliadas, foram selecionadas as melhores estimativas de parâmetros $\mathrm{v}_{\mathrm{j}}$, $\mathrm{h}_{\mathrm{j}}$ e $\mathrm{g}_{\mathrm{i}}$ para os experimentos topcrosses, com maior ênfase para os $\mathrm{g}_{\mathrm{i}}$ dos caracteres PG e CE, para o experimento I, e PG para o experimento II. No experimento I, foram selecionadas, para predição de médias de compostos, as populações UEL SI, UEL PAP, UEL ZP, UEL PP, CMS 43 e RS 20. Para as condições do experimento I, rejeitaram-se as populações UEL MPS e UEL PAG em razão dos valores negativos de gi para o caráter PG, apesar de seus valores positivos de $g_{i}$ para CE. No experimento II, foram selecionadas as populações UEL PAG e CMS 42. Miranda Filho \& Chaves (1991) salientam que a variabilidade genética de um composto está relacionada à diversidade genética entre as variedades que entram em sua formação.

Na Tabela 6, são apresentadas as médias de compostos de tamanho k estimadas para os caracteres estudados. Para o caráter PG, os grupos de compostos de

Tabela 6. Médias individuais de compostos de milho pipoca de diferentes tamanhos (k) estimadas a partir das médias individuais das 14 populações e seus respectivos topcrosses ${ }^{(1)}$.

\begin{tabular}{|c|c|c|c|c|c|c|c|c|c|c|c|c|}
\hline \multicolumn{7}{|c|}{ Experimento I $^{(2)}$} & \multicolumn{6}{|c|}{ Experimento $\mathrm{II}^{(3)}$} \\
\hline $\mathrm{k}=2$ & $\mathrm{PG}$ & $\mathrm{CE}$ & NEP & $\mathrm{AP}$ & PREP & $\mathrm{FF}$ & $\mathrm{k}=2$ & PG & NEP & $\mathrm{AP}$ & PREP & $\mathrm{FF}$ \\
\hline 12 & 3,01 & 24,0 & 0,91 & 1,91 & 0,61 & 59 & 12 & 2,14 & 1,30 & 1,53 & 0,57 & 66 \\
\hline 13 & 3,12 & 23,4 & 1,05 & 1,96 & 0,62 & 58 & 13 & 2,10 & 1,21 & 1,45 & 0,57 & 65 \\
\hline 14 & 3,90 & 24,1 & 1,13 & 2,14 & 0,62 & 58 & 14 & 2,08 & 1,10 & 1,62 & 0,59 & 69 \\
\hline 15 & 2,87 & 24,5 & 1,00 & 1,81 & 0,61 & 55 & 15 & 2,16 & 1,29 & 1,61 & 0,56 & 63 \\
\hline 23 & 2,83 & 24,7 & 1,05 & 1,95 & 0,63 & 59 & 23 & 2,24 & 1,29 & 1,45 & 0,54 & 62 \\
\hline 24 & 3,61 & 25,3 & 1,13 & 2,14 & 0,63 & 58 & 24 & 2,22 & 1,18 & 1,62 & 0,57 & 67 \\
\hline 25 & 2,58 & 25,8 & 1,00 & 1,81 & 0,61 & 56 & 25 & 2,30 & 1,37 & 1,61 & 0,53 & 61 \\
\hline 34 & 3,72 & 24,7 & 1,27 & 2,18 & 0,64 & 58 & 34 & 2,18 & 1,09 & 1,54 & 0,57 & 66 \\
\hline 35 & 2,69 & 25,2 & 1,14 & 1,85 & 0,62 & 55 & 35 & 2,26 & 1,28 & 1,53 & 0,53 & 60 \\
\hline 45 & 3,46 & 25,9 & 1,22 & 2,04 & 0,62 & 55 & 45 & 2,24 & 1,17 & 1,70 & 0,55 & 64 \\
\hline $\mathrm{k}=3$ & & & & & & & $\mathrm{k}=3$ & & & & & \\
\hline 123 & 3,23 & 23,6 & 1,01 & 1,96 & 0,63 & 59 & 123 & 2,27 & 1,32 & 1,48 & 0,57 & 65 \\
\hline 124 & 3,81 & 24,0 & 1,07 & 2,11 & 0,62 & 58 & 124 & 2,23 & 1,24 & 1,59 & 0,58 & 68 \\
\hline 125 & 3,11 & 24,8 & 0,98 & 1,86 & 0,61 & 57 & 125 & 2,26 & 1,38 & 1,59 & 0,55 & 63 \\
\hline 134 & 3,87 & 23,5 & 1,17 & 2,13 & 0,63 & 58 & 134 & 2,22 & 1,15 & 1,53 & 0,58 & 67 \\
\hline 135 & 3,19 & 24,3 & 1,08 & 1,88 & 0,62 & 57 & 135 & 2,25 & 1,29 & 1,52 & 0,55 & 62 \\
\hline 145 & 3,76 & 24,7 & 1,14 & 2,02 & 0,62 & 56 & 145 & 2,21 & 1,21 & 1,64 & 0,57 & 65 \\
\hline 234 & 3,66 & 24,7 & 1,16 & 2,13 & 0,64 & 58 & 234 & 2,33 & 1,23 & 1,54 & 0,57 & 65 \\
\hline 235 & 2,96 & 25,4 & 1,07 & 1,89 & 0,63 & 57 & 235 & 2,35 & 1,37 & 1,54 & 0,53 & 60 \\
\hline 245 & 3,53 & 25,8 & 1,12 & 2,03 & 0,62 & 57 & 245 & 2,32 & 1,29 & 1,65 & 0,55 & 63 \\
\hline 345 & 3,61 & 25,4 & 1,22 & 2,05 & 0,63 & 56 & 345 & 2,31 & 1,19 & 1,59 & 0,55 & 62 \\
\hline $\mathrm{k}=4$ & & & & & & & $\mathrm{k}=4$ & & & & & \\
\hline 1234 & 3,79 & 23,7 & 1,11 & 2,10 & 0,63 & 58 & 1234 & 2,31 & 1,26 & 1,54 & 0,58 & 67 \\
\hline 1235 & 3,26 & 24,5 & 1,04 & 1,91 & 0,63 & 58 & 1235 & 2,32 & 1,36 & 1,53 & 0,55 & 62 \\
\hline 1245 & 3,71 & 24,8 & 1,08 & 2,02 & 0,62 & 57 & 1245 & 2,29 & 1,30 & 1,62 & 0,57 & 64 \\
\hline 1345 & 3,77 & 24,4 & 1,17 & 2,03 & 0,63 & 57 & 1345 & 2,29 & 1,22 & 1,57 & 0,57 & 64 \\
\hline 2345 & 3,59 & 25,3 & 1,15 & 2,04 & 0,63 & 57 & 2345 & 2,37 & 1,29 & 1,58 & 0,55 & 62 \\
\hline $\mathrm{k}=5$ & & & & & & & $\mathrm{k}=5$ & & & & & \\
\hline 12345 & 3,71 & 24,5 & 1,11 & 2,03 & 0,63 & 57 & 12345 & 2,34 & 1,30 & 1,57 & 0,56 & 64 \\
\hline
\end{tabular}

(1)PG: peso de grãos (t ha $\left.{ }^{-1}\right)$; CE: capacidade de expansão ( $\mathrm{mL} \mathrm{mL}^{-1}$ ); NEP: número de espigas por planta; AP: altura de planta (m); PREP: posição relativa da espiga na planta (m); FF: florescimento feminino (dias). (2)1: UEL SI; 2: UEL ZP; 3: UEL PP; 4: CMS 43; 5: RS 20. (3)1: UEL MP; 2: UEL PAG; 3: UEL PP; 4: CMS 42; 5: CMS 43. 
mesmo tamanho com maior $\mathrm{k}(\mathrm{k}=4 \mathrm{e} \mathrm{k}=5)$ apresentaram tendência de melhores médias, em relação aos grupos de menor $k(k=2$ e $k=3)$. Esse fato foi também observado por Miranda Filho \& Chaves (1991), em trabalho voltado à predição de médias de compostos de milho.

Comparando-se a média de PG e CE do composto amplo com as médias gerais de PG e CE dos demais compostos, no experimento I, verificou-se que $\mathrm{C}_{\mathrm{T}} 12345$ apresentou menor média. No entanto, essas diferenças $(<0,1)$ não confirmaram que a superioridade dos compostos menores pudesse justificar sua escolha, em detrimento do composto $\mathrm{C}_{\mathrm{T}} 12345$; o mesmo ocorreu para o experimento II (Tabela 6). Sob o ponto de vista do melhoramento de plantas, valores de $\mathrm{k} \geq 4$ devem ser adotados na maioria dos casos (Miranda Filho \& Chaves, 1991).

O composto $\mathrm{C}_{\mathrm{T}} 12345$ foi selecionado, entre os demais, pelos seus valores de PG e CE. A utilização do $\mathrm{C}_{\mathrm{T}} 12345$, como população base no melhoramento, permitiria a exploração de seu potencial de variabilidade e de elevada freqüência de alelos favoráveis para PG e CE.

\section{Conclusões}

1. A metodologia de cruzamento topcrosses intra-grupo é eficiente para auxiliar na escolha de populações para formar compostos.

2. O testador utilizado possibilita a identificação de genótipos superiores, entre as populações avaliadas.

3. A característica PG é influenciada tanto por efeitos aditivos como por efeitos de dominância.

4. As populações UEL SI, UEL ZP, UEL PP, CMS 43 e RS 20 apresentam-se mais promissoras para a síntese de compostos produtivos e com qualidade comercial.

\section{Agradecimentos}

Ao CNPq e à CAPES, pelas bolsas de estudo concedidas.

\section{Referências}

CARPENTIERI-PÍPOLO, V.; TAKAHASHI, H.W.; ENDO, R.M.; PETEK, M.R.; SEIFERT, A.L. Correlações entre caracteres quantitativos em milho pipoca. Horticultura Brasileira, v.20, p.551-554, 2002.
CHAVES, L.J.; MIRANDA FILHO, J.B. de. Predicting variety composite means without diallel crossing. Brazilian Journal of Genetics, v.20, p.501-506, 1997.

CRUZ, C.D.; REGAZZI, A.J. Modelos biométricos aplicados ao melhoramento genético. 2.ed. Viçosa: UFV, 1994. 390p.

EMBRAPA. Centro Nacional de Pesquisa de Milho e Sorgo (Sete Lagoas, MG). Recomendações técnicas para o cultivo do milho. 2.ed. Brasília: Embrapa-SPI; Sete Lagoas: Embrapa-CNPMS, 1996. 204p.

FERREIRA, J.M. Análise genética de populações visando resistência à ferrugem (Puccinia polysora Underw.) em milho (Zea mays L.). 1999. 230p. Tese (Doutorado) - Escola Superior de Agricultura Luiz de Queiroz, Piracicaba.

GALVÃO, J.C.C.; SAWAZAKI, E.; MIRANDA, G.V. Comportamento de híbridos de milho-pipoca em Coimbra, Minas Gerais. Revista Ceres, v.47, p.201-218, 2000.

GARDNER, C.O. Simplified methods for estimating constants and computing sums of square for a diallel cross analysis. Fitotecnia Latinoamericana, v.4, p.1-12, 1967.

GÖKMEN, S. Effects of moisture content and popping method on popping characteristics of popcorn. Journal of Food Engineering, v.65, p.357-362, 2004.

HALLAUER, A.R. Methods used in developing maize inbreds. Maydica, v.35, p.1-16, 1990.

INSTITUTO AGRONÔMICO DE CAMPINAS. Recomendações de adubação e calagem para o Estado de São Paulo. 2.ed. Campinas: IAC, 1996. 285p. (Boletim técnico, 100).

LIRA, M.A. Seleção entre e dentro de famílias de meios-irmãos para produção, capacidade de expansão e correlações entre alguns caracteres em milho pipoca (Zea mays L.). 1983. 63p. Dissertação (Mestrado) - Universidade Federal de Lavras, Lavras.

LONNQUIST, J.H.; LINDSEY, M.F. Topcross versus $\mathrm{S}_{1}$ line performance in maize. Crop Science, v.4, p.580-584, 1964.

LYERLY, P.J. Some genetic and morphologic characters affecting the popping expansion of popcorn. Journal of the American Society of Agronomy, v.34, p.986-999, 1942.

MIRANDA FILHO, J.B. de; CHAVES, L.J. Procedures for selecting composites based on prediction methods. Theoretical and Applied Genetics, v.81, p.265-271, 1991.

PACHECO, C.A.P. Ensaio nacional de milho pipoca: resultados do ano agrícola de 1991/1992. Sete Lagoas: Embrapa-CNPMS, 1992. 37p.

PEREIRA, M.G.; AMARAL JÚNIOR, A.T. Estimation of genetic components in popcorn based on nested design. Crop Breeding and Applied Biotechnology, v.1, p.3-10, 2001.

PINTO, R.J.B.; SCAPIM, C.A.; FERREIRA NETO, A.; PACHECO, C.A.P.; ROYER, M.; PEDRONI, M.V.; SALVADORI, R.K.; SILVA, R.M. Analysis of testers with broad and narrow genetic base for topcrosses in popcorn breeding. Crop Breeding and Applied Biotechnology, v.4, p.152-162, 2004. 
REGITANO NETO, A. Avaliação quantitativa da estrutura genética de uma população de milho pipoca. 1993. 115p. Dissertação (Mestrado) - Escola Superior de Agricultura Luiz de Queiroz, Piracicaba.

SANTACRUZ-VARELA, A.; WIDRLECHNER, M.P.; ZIEGLER, K.E.; SALVADOR, R.J.; MILLARD, M.J.; BRETTING, P.K. Phylogenetic relationships among North American popcorns and their evolutionary links to Mexican and South American popcorns. Crop Science, v.4, p.1456-1467, 2004.

SAS INSTITUTE (Cary, Estados Unidos). SAS language and procedures: usage statistics SAS Institute. Version 6. Cary, 1995. 373p.

SAWAZAKI, E. A cultura do milho pipoca no Brasil. O Agronômico, v.53, p.11-13, 2001.

SAWAZAKI, E. Melhoramento do milho pipoca. Campinas: Instituto Agronômico, 1995. 21p.
SAWAZAKI, E. Parâmetros genéticos em milho pipoca. 1996. 157p. Tese (Doutorado) - Escola Superior de Agricultura Luiz de Queiroz, Piracicaba.

SILVA, W.J. da; VIDAL, B.C.; MARTINS, M.E.Q.; VARGAS, H.; PEREIRA, A.C.; ZERBETTO, M.; MIRANDA, L.C.M. What makes popcorn pop. Nature, v.362, p.417, 1993.

SOUZA, E.D. Divergência genética e avaliação de famílias $S_{1}$ e top crosses de milho, utilizando-se caracteres agronômicos e marcadores RAPD. 2000. 88p. Tese (Doutorado) - Universidade Federal de Lavras, Lavras.

VENCOVSKY, R. Alguns aspectos teóricos e aplicados relativos a cruzamentos dialélicos de variedades. 1970. 59p. (Livre Docência) - Escola Superior de Agricultura Luiz de Queiroz, Piracicaba.

VENCOVSKY, R.; BARRIGA, P. Genética biométrica no fitomelhoramento. Ribeirão Preto: Sociedade Brasileira de Genética, 1992. 486p.

Recebido em 18 de março de 2005 e aprovado em 11 de novembro de 2005 